\title{
Exploration of Creative Landscape Design Based on Chinese Traditional Gardening Techniques
}

\author{
Aibin Yan ${ }^{1, a}$, Ling Yang $2, b$ \\ ${ }^{1}$ East China University of Science and Technology, 130 Meilong Road, Shanghai, China \\ Tongji University Shanghai Institute of Design and Innovation, 1249 Siping Road, Shanghai, China \\ 2 East China University of Science and Technology, 130 Meilong Road, Shanghai, China \\ a yanaib@126.com, b1196915292@qq.com
}

\begin{abstract}
Keywords: traditional garden; gardening techniques; scenic image; scenic view; scenic guidance; creative landscape space;

Abstract: In recent years, with the growing attention to Chinese own cultural characteristics, how to create a unique cultural landscape space has increasingly become one of the most important topics in landscape architecture, especially in the urgent need of activation and renewal in historical areas, scenic construction, cultural landscape. While the use of traditional gardening techniques is one of the most effective ways and methods, particularly using Jiangnan classical gardening approach in the design and build of new creative landscape. This article gives multifaceted exploration and analysis on how to use the traditional gardening techniques for creative landscape, and carries out a more systematic combing from scenic constituents, scenic organization, scenic structure and scenic guidance, in accordance with the analysis and research methods on scenic image of classical garden.
\end{abstract}

\section{Introduction}

In recent years, as all sectors of the society and the industry pay more attention to Chinese own cultural characteristics, how to create a unique cultural landscape, how to create a unique cultural landscape space has increasingly grown into one of the most important topics in landscape architecture, especially in the urgent need of activation and renewal in historical areas, scenic construction, cultural buildings and landscape. Recently, some outstanding design works, such as I.M.Pei's Suzhou Museum; Li Xinggang's JiXi Museum; Wang Shu's Waterfront Hill, China Academy of Arts and Gongwang Art Museum; Li Li's Fei Xiaotong Memorial Hall, Jiangcun; Sifenyuan of Hua Li Office; Huaxin Exhibition Center of Scenic Architecture; Li Xiaodong's Miaolu, Lijiang, etc., are innovative works created after deep understanding of classical gardening theory and techniques, in which the design ideas and methods are worthy of careful and systematic summary combing.

\section{Chinese classical gardening techniques and its contemporary significance}

Study on landscape design of Jiangnan Classical garden.Jiangnan garden, as a boutique art of time and space in Chinese classical garden, has made a high artistic achievements. During each dynasty, there is no lack of relevant research on systematic theories and methods in shaping of ground surface, planning of architectural elements, planning of vegetation, creation of scenic imagery, concealing and borrowing of scenes and other aspects.

For example, "Yuan Ye" (a book of garden making) written by late Ming Dynasty garden designer Ji Cheng, is the first treatise that improving garden practice to the theory. The word "Though made by mankind, it looks natural formed" and "clever in Jie Jing(borrowing of scenes), excellent in fitness" is his pithy summary of Chinese garden art. In contemporary research results, "A Treatise On The Garden Of Jiangnan" by Yang Hongxun, is the best in grand and rigorous system, scientific and concise elaboration, deeply and systematically expounding the art theory and creation theory of Chinese classical garden, in-depth appreciating the typical Jiangnan gardens. The book takes scene 
imagery as the main line and systematically analyzes the traditional garden from the perspective of subject research, which is of great significance for the study of Jiangnan traditional garden and the method of refining garden. The research combing of this paper will mainly follow the scene imagery creation theory of Yang Hongxun's “A Treatise On The Garden Of Jiangnan”.

Study on the application of classical gardening techniques in Modern Landscape. In the past three decades, landscape design profession in China was to follow the West, directly taking the form, copying and imitating, completely ignoring national conditions of China, leading to several errors such as lagging views, unclear concepts, formalism and over publicized in domestic landscape design for a long time. With the proposition of landscape localization, people look back and gradually discovered the great value and guiding significance of Chinese traditional garden design techniques. The traditional landscape design approach is the root of creatinng local environment, adapting to the current pursuit of harmony between man and nature. Presently, there is some good application exploration of traditional classical gardening techniques in modern landscape design in China, as well as some successful practice cases. The living environment of the new Chinese landscape, combining traditional design with innovation, such as Wanke Fifth Park, Lucheng Tao Li Chun Feng, etc., has been widely recognized and made a positive attempt to the application of traditional garden approach in modern landscape.

\section{The Theory and Method of Scenic Imagery Creation in Traditional Gardens}

Scenic Image. A scenic image is the basic unit in the art of garden.It is a spatial concept, comprising of scenic constituents and scenic guidance. Scenic constituents including natural and artificial constituents are the material foundation of garden constructing. Scenic guidance, with a focus on space. regarded as the key to garden's aesthetic value and functional value, is an organizing factor that represents the structural relationship of the garden. Therefore, scenic image is the co-ordination of constituents and guidance. Researching the traditional garden from the perspective of scenic imagery creation is a systematic scientific method, having a positive practical significance on the analysis of classical garden space and guidance of landscape design.

Scenic Constituents. Scenic constituents mainly include building earth mound and stacking stones, management of water surface, planning of vegetation and planning of architectural elements. Mountain and water features contribute to the shaping of ground surface, while vegetation planning and architectural planning are more of spatial shaping. In addition to these four elements, other elements like animals are often used to create a characteristic space.

Scenic Guidance. Guidance is an organizing factor that represents the structural relationship of the garden. The key lies in guiding during the creating of garden. Guidance also determines the structural relationship of the sceneries, showing the changes of imagery and scenery. Scenic guidance as medium links visitors to the garden. It is the scenic guiding enables visitors to achieve their garden activities and immersive feelings. Scenic guidance including route for dynamic views and base point for static views, of which twists and turns of route can best reflect the feature of Chinese classical garden: getting wide imagery from the small view, seeing varied scenery while strolling.

Scenic Switching. A variety of techniques are used to achieve the switching of scenes, adding changes to garden space. Scenic switching methods involve concealing of scenes, revealing of scenes, contraposition of scenes, borrowing of scenes, etc.. Concealing and revealing of scenes is often taken in combination to achieve the purpose of scene separation and transition. Scenic contraposition often used in the turning point of routes and the changes of scenes, together with scenic concealing basically decide the form of guidance, having a relationship of mutual transformation. Scenic borrowing is the most important technique in garden making, which can be summarized as "Yuan Jie"(borrowing from afar) and "Lin Jie"(borrowing from near-by" in the space, regarded as" "Yin-Shi-Er-Jie"(borrowing according to the change of time) in the time dimension, reflecting the characteristics of an art of time and space in landscape. 
The creation of scenic imagery. The evaluation standard of Chinese classical garden art is based on the creation of "Yi Jing"(scenic imagery purporting the non-material and intangible quality of a scenic image or garden space that is capable of evoking an aesthetic atmosphere or emotional appeal). There are many necessary conditions for the formation of Yi Jing. First, following the laws and principles of the natural ecologic world and reflecting the harmonious relationship between man and nature. Secondly, garden maker and visitor (the owner) should reach consensus in mind, usually having a similar cultural background and ideology, and finally get the effect of limited scenery but infinite imagery.

\section{Analyzing the method of planning creative landscape space by using scenic imagery theory}

The creation and innovation application of Scenic constituents. (a) Building earth mound and stacking stones. In recent years, there have been many new landscape continuing to create space intention by building earth mound and stacking stones, which is usually used in traditional garden. Fig. 1. Shows the Rocky Mountain in I.M.Pei's Suzhou Museum, which is one of the outstanding cases. Simple founder blocks of plain concrete are composed to express the abstract intention of mountain as shown in Fig. 2 in Li Xinggang's Jixi Museum. Tiles are used to create the image of mountain in some residential houses. In a house of Xinchang Old Town, a innovation based on the Chinese traditional garden skill named "Qiao-Bi-Shan" is found. Fig. 3 shows the innovation of "Bi-shan". It is a painted wall combined with stone and rockery, creating a new rockery landscape described as both virtual and real.

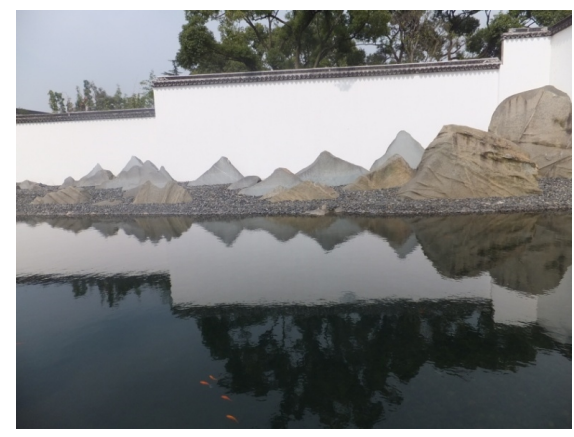

Fig. 1. The Rocky Mountain in Suzhou Museum (Self-photographing)

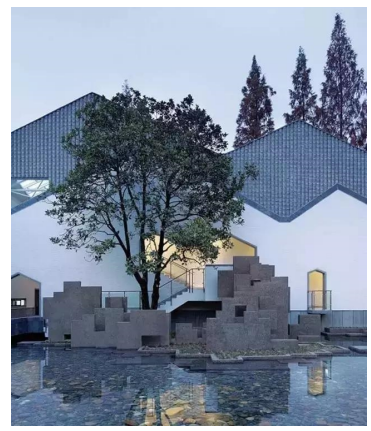

Fig. 2. Jixi Museum (image source: http://www.ikuku.cn/ post/40258)

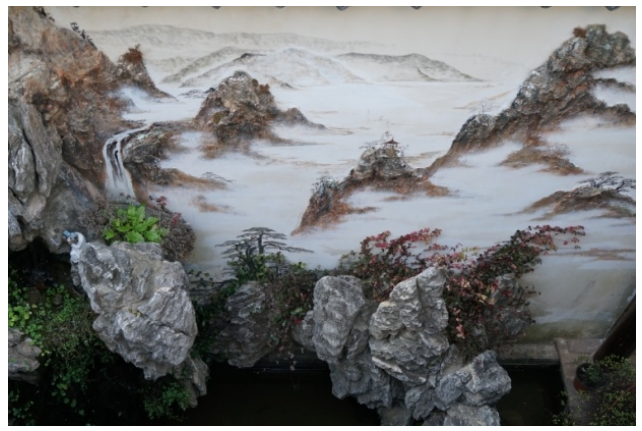

Fig. 3. The innovation of "Bi-Shan" in Xinchang Old Town (Self-photographing)

(b) Management of water surface. Learning from Chinese classical garden making skill named "Han-Yuan-Shui-Zuo"( creating landscape to symbolize the water view with constituents instead of water ), "dry wadis", usually filled with cobblestone and niuxin stone, are designed as water landscape in recent years. It is similar to "Karesansui", a gardening method from Japan. The water landscape in Suzhou Museum is a successful case. Cobblestones are placed from the bottom of stone mountain into the water, forming a natural transition and extension from rockery to water surface.

(c) Vegetation. Learning the planning of vegetation from classical garden can often create a fresh and brief landscape. Fig. 4 shows the Nan Lian Garden in front of the Chi Lin Nunnery, which is a successful example. Planning of vegetation, a scale control technique of classical gardening is fully used to form a serene elegant and wide landscape environment through small one.

(d) Architecture. To create new landscape under the principles and methods that garden architecture should be placed appropriately. In Li Xinggang's Jixi Museum using traditional building materials - tiles to form "Tile Wall" shown in fig. 5, is a representative case. 


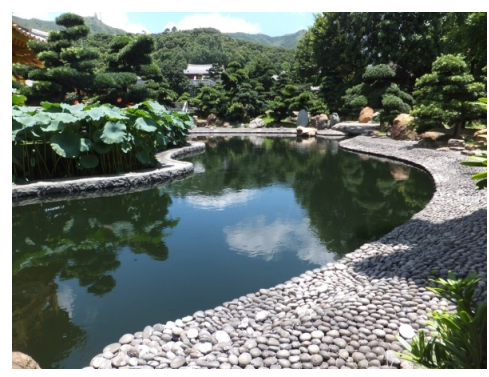

Fig. 4. The Nan Lian Garden in front of the Chi Lin Nunnery (Self-photographing)

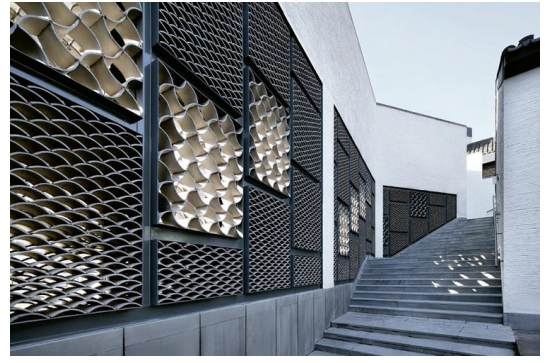

Fig. 5. Jixi Museum (image source: http://www.ikuku.cn/post/40258)

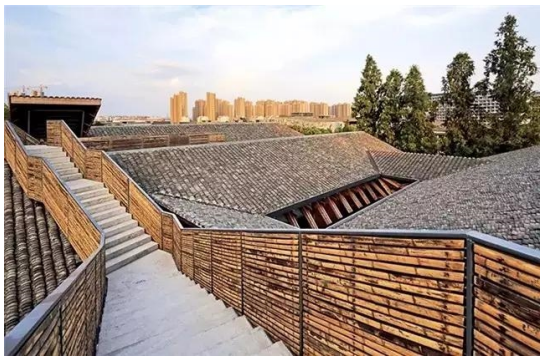

Fig. 6. Waterfront Hill, China Academy of Arts (Self-photographing)

Scene Organizational Approach and Its Innovative Application. Scenic view is the basic unit of scenic imagery organized by scenic constituents, which is two-dimensional and static. The center area in Suzhou Museum is the excellent case of scene organization. A main attraction combined of stone mountain, lake-type water and folding bridge opposites to the main entrance hall, forming a succinct and peaceful space.

The composition of scenic imagery and its innovative use. The composition of the scenic imagery refers to the spatial combination of static "scenic view" in Chinese classical gardens, which is the core content of classical gardening techniques and the easier method of shaping traditional space characteristics in the modern landscape design and construction. But excellent works are rare in this area.

Wang Shu's Waterfront Hill, China Academy of Arts and Gongwang Art Museum shown in fig.6; Li Xinggang's JiXi Museum; Li Li's Fei Xiaotong Memorial Hall, Jiangcun; Huaxin Exhibition Center of Scenic Architecture; Sifenyuan of Hua Li Office shown in fig.7, etc., are cases successfully using the technique of scenic composition of classical garden and traditional architecture. Building earth mound and stacking stones, management of water surface, planning of vegetation and planning of architectural elements are integrated used to the construction of Three - dimensional Space. Fig. 8 and fig.9 show the plan and inner court space of Fei Xiaotong Memorial Hall, Jiangchun.

This method is helpful to promote the creation of new landscape in rich of Chinese culture and characteristic.

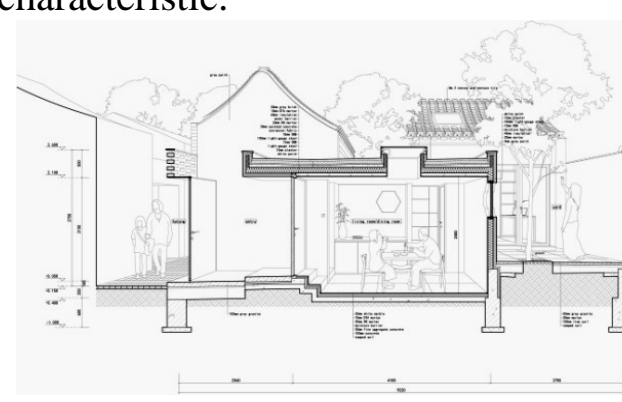

Fig. 7. Sifenyuan (image source: http://www.gooood.hk/split-courtya rd-house-beijing-china-by-tao.htm)

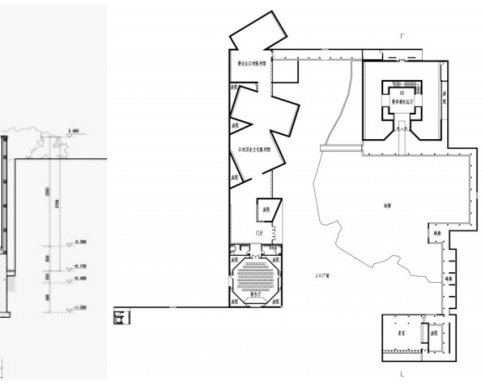

Fig. 8. Plan of Fei Xiaotong Memorial Hall, Jiangchun

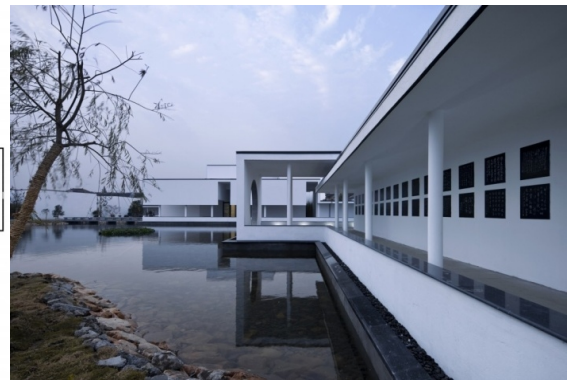

Fig. 9. The inner court space of Fei Xiaotong Memorial Hall, Jiangchun (image source: http://www.ikuku.cn/project/feixiaotongjiangcun-jinianguan-lili)

Scenic Guidance and Its Innovative Application. Scenic guidance is a relationship of switching different scenes, similar to the film technique - montage. Including two aspects: route and concealing and revealing, scenic guidance, a real approach of leading garden space from three to four dimensions, is an art of time and space. Li Xinggang's JiXi Museum; Li Li's Fei Xiaotong Memorial Hall, Jiangchun; Sifenyuan of Hua Li Office are good cases in reflecting the scenic guiding techniques and four-dimensional garden art. 
Nara National Museum, designed by Junzo Yoshimura, Japan, is an example of using scenic guidance to create new space, in which relationship of guiding, concealing and revealing that learnt from Shugakuin Imperial Villa, stroling water court with pound of Ryoanji can be found.

\section{Conclusions}

It is worthwhile to explore new ideas and new methods for creative landscape space design and construction by learning from traditional gardening techniques. Study can start from the analysis of scenic imagery, so as to guide the rich diversity of landscape and the ecological environment of human settlements. Then deeply analyzing the organization of garden path, the changeability of route, the setting of base point, the switching of scenic image, the creation of scenic imagery, etc., and creating scenic image by specific techniques such as concealing of scenes, contraposition of scenes, borrowing of scenes, etc.. In addition, the design should focus on the sustainable development of ecology and context, pay attention to the renewal and extension of the place spirit.

Traditional techniques of making a garden as an effective way to create imagery and scenery should be more widely used. Traditional gardening is the essence of Chinese garden art. For a long time, People's understanding of it only stay in the copy of elements and ignoring its utility as a design method. By scientific analyzing of gardening method, the unique view of the ancients in space construction can be learned, having great guiding significance in modern landscape design.

In existing studies, the emergence of the new Chinese landscape is an interpretation of the traditional gardening techniques to a certain degree. Although techniques like concealing of scenes, contraposition of scenes, borrowing of scenes are skilled using in new Chinese landscape, but it did not get rid of the landscape style of white walls and black tiles and is still a copy of elements to a certain extent. Taking scenic imagery as analytical focus in landscape construction may lead to the wider use of traditional gardening techniques. This art of time and space can guide the ecological environment of human settlements and perhaps lead the way of urban renewal to sustainable development.

\section{Acknowledgements}

Supported by the National Natural Science Foundation of China(NSFC) (51478185), and Shanghai Summit Discipline in Design(DB17030).

\section{References}

[1] Hongxun Yang. A Treatise On The Garden Of Jiangnan [M]. Beijing: China Architecture \& Building Press (2011). (In Chinese).

[2] Cheng Ji. The explanation of Yuan Ye [M]. Beijing: China Architecture \& Building Press (1988) . (In Chinese).

[3] Christian Norberg-Schulz. Towards A Phenomenology Of Architecture [M]. Wuhan: Huazhong University of Science and Technology Press (2010) . (In Chinese). 